\title{
COMUNICACIÓN CULINARIA EN REPÚBLICA DOMINICANA: TECNOLOGÍA, CHEFS, FOOD STYLISTS Y FOODIES
}

\author{
Maricha Martínez Sosa ${ }^{\mathrm{a} 1}$, Lucero Liriano ${ }^{\mathrm{a} 2}$, Georgina Batista Schrils ${ }^{\text {a3 }}$ \\ ${ }^{a}$ República Dominicana \\ a1 info@marichams.com, a1 Lucero.liriano@gmail.com, ${ }^{a 1}$ gbatista1903@gmail.com
}

\begin{abstract}
Technological development has transformed what we eat and how we enjoy it. Gastronomy has evolved, and this is reflected in the progressive use of innovative formats and communication platforms, which have also influenced the rituals of food socialization. The popularity of new media has led to an increase in the creation of gastronomic content in the Dominican Republic. In order to address it, this research has been carried out taking two axes as a point of analysis: the bibliography on the food heritage of this Caribbean country and the profiles of subjects, brands, and initiatives that serve as digital ambassadors of this culinary culture and use the networks to generate impact on society.
\end{abstract}

Keywords: chefs, communication, culinary, Dominican Republic, food stylists, foodies, gastronomy, technology.

Resumen: El desarrollo tecnológico ha transformado qué comemos y cómo lo disfrutamos. La gastronomía ha evolucionado y esto se refleja en el uso progresivo de innovadores formatos y plataformas de comunicación, que han influenciado también los rituales de socialización de los alimentos. La popularización de los nuevos medios ha propiciado que la creación de contenido gastronómico en la República Dominicana aumente. Para abordarla se ha hecho la esta investigación tomando como punto de análisis dos ejes: la bibliografía en torno al patrimonio alimentario de este país caribeño y los perfiles de sujetos, marcas e iniciativas que fungen como embajadores digitales de dicha cultura culinaria y se valen de las redes para generar impacto en la sociedad.

Palabras clave: chefs, comunicación, culinaria, República Dominicana, food stylists, foodies, gastronomía, tecnología.

Citar como: Martínez Sosa, M., Liriano, L., Batista Schrils, G. (2022). "Comunicación culinaria en República Dominicana: tecnología, chefs, food stylists y foodies". En: Actas del III Congreso Internacional sobre Patrimonio Alimentario y Museos. 25-26 noviembre, 2021, Valencia, España. pp. 263-282. https://doi.org/10.4995/EGEM2021.2021.13408 


\section{Introducción}

Mucho se ha cambiado desde que la transmisión y supervivencia del acervo culinario recaía sobre el formato oral. El desarrollo tecnológico ha transformado qué comemos y cómo lo disfrutamos, influenciando los rituales de socialización de los alimentos. La gastronomía en República Dominicana también ha evolucionado, esto se refleja en el uso progresivo de innovadores formatos y plataformas de comunicación. Aquellos que han elegido las artes culinarias como profesión cuentan con nuevos aliados. Desde los "reality shows" en los que amateurs muestran su proeza en la cocina ante las cámaras por la victoria, hasta quienes retocan los alimentos detalladamente. Tecnologías y perfiles antes inexistentes, narran la historia de recetas, experiencias de su consumo y proveniencia de sus ingredientes. Entre ellos encontramos categorías como food stylists, que resaltan la esencia de los platos, y foodies que buscan magníficas creaciones.

Luego de presentar una selección de la bibliografía gastronómica en la República Dominicana, este documento enlista sujetos que se han abierto camino en este sector. A través del análisis de la literatura existente se pretende iniciar la recolección del trabajo e identificación de perfiles de abanderados y embajadores digitales de la gastronomía que más allá de los fogones, están detrás de los libros, programas y dispositivos.

\section{Objetivos}

\section{- Objetivo General}

Identificar perfiles de abanderados y embajadores de la gastronomía que engrandecen la calidad culinaria de la República Dominicana.

\section{- Objetivos Específicos}

- Presentar la evolución de la alimentación en la República Dominicana, su entorno y plataformas actuales para la comunicación gastronómica.

- Identificar los principales actores del ecosistema comunicacional dominicano en materia culinaria.

- Definir las nuevas ramas profesionales, así como las herramientas que las impulsan.

- Compilar parte de la literatura y publicaciones existentes sobre gastronomía dominicana.

\section{Desarrollo de la Innovación}

El presente documento analiza el ecosistema comunicacional gastronómico: antecedentes, bibliografía y principales actores en los medios digitales.

\subsection{Antecedentes}

Los elementos que componen la identidad cimentan las raíces sociales de los pueblos "a través de los imaginarios simbólicos compuestos por tradiciones, lenguas, hábitos, gastronomía, celebraciones, rituales y expresiones artísticas, entre otros. Para su construcción, como 
definición social, se requiere una conciencia histórica y un proceso de valoración colectiva" (Martínez Sosa, 2020).

La gastronomía, arte de la buena preparación y relación de los alimentos, ha evolucionado a través de los años como resultado de las diferentes interacciones sociales, culturales y migratorias que impactaron el devenir histórico, y por consecuencia culinario, de los pueblos. Ejemplo de ello es la República Dominicana, cuyo menú ofrece mezclas culturales, sumadas a la diversidad de climas y ecosistemas.

Gracias a investigadores como Marcio Veloz Maggiolo, se ha recapitulado la información histórica y científica disponible acerca de la gastronomía indígena. Los taínos, primeros habitantes de la isla, basaron su alimentación en el cultivo de yautía, auyama, mapuey, yuca, guanábana, jobos, entre otros. Lo que pescaban era complementado con preparaciones como el casabe de yuca, elaborado con utensilios como el guayo y el cibucán (especie de colador); y mabí, una bebida fermentada. Obras como las de Hugo Tolentino Dipp permiten entender qué sucedió con la alimentación a partir de la llegada de los españoles (1492) con la introducción de técnicas, tradiciones, alimentos típicos e ingredientes que se volverían fundamentales, como el arroz, los plátanos y la carne ganadera.

El sazón que hoy nos caracteriza llegó con los africanos, quienes vinieron como esclavos y trajeron jengibre, molondrón, malagueta y otras especias, así como la técnica de sofreír ajo y cebolla. Otros grupos con aportes culinarios fueron los cocolos de las Antillas Menores, los árabes e italianos. Estos procesos migratorios y las importaciones facilitadas por la globalización de los mercados han resultado en un variado menú, con trazas de sincretismo cultural, que se ha integrado en las tradiciones y cotidianidad dominicana.

\subsection{Gastronomía dominicana, hoy}

Al igual que diferentes elementos de esta cultura, la culinaria ha sido el resultado de las circunstancias y necesidades de la población. Según el portal RutaGourmet.do, es un fenómeno:

\footnotetext{
Esencial de nuestro patrimonio cultural inmaterial que se crea y transforma en cada bocado, en cada cocción, en cada aroma, llena del sabor de lo que somos, de nuestro origen. Es una cocina sabrosa, que invita siempre a la celebración. Se crea y recrea un espacio común, un mapeo colectivo que refleja nuestras costumbres y tradiciones culinarias, utilizando como base aspectos culturales e históricos del país.
}

Características de la gastronomía dominicana son su variedad, practicidad y resiliencia. Se distinguen técnicas artesanales, algunas heredadas de los taínos, nuestros primeros artesanos, quienes elaboraban manualmente sus herramientas. Hoy, hay quienes se identifican como artesanos gastronómicos por sus conocimientos de comida e ingredientes criollos, por desarrollar innovaciones en el área, ser reconocidos por su experiencia y tener vocación y aptitud por la cocina dominicana (López, 2021).

La diversidad de platos típicos incluye "la bandera" (arroz blanco, habichuelas guisadas y carne); sancocho (sopa de distintas carnes y tubérculos); mangú (puré de plátanos verdes, acompañado con rodajas de queso, salami o huevo); morir soñando (bebida de leche con jugo de naranja), entre otros. 

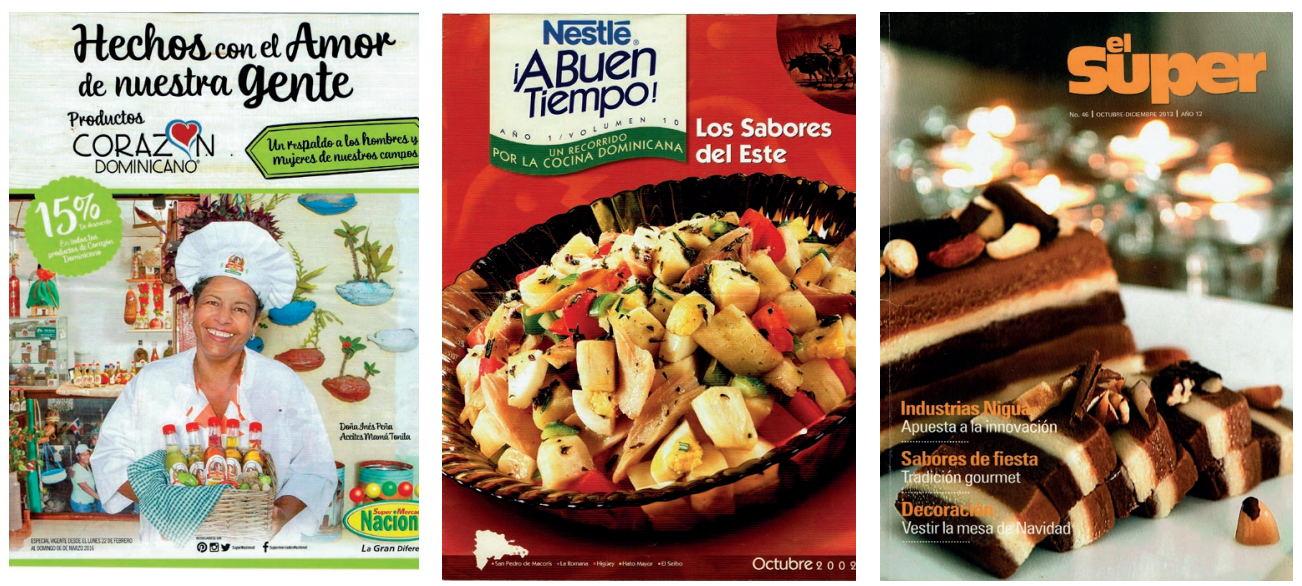

Figura 1. Publicaciones gastronómicas dominicanas, parte de una colección privada.

Fuente: Biblioteca de Isis Mercedes Flaquer.

\subsection{Comunicación, tecnología y gastronomía}

La tecnología ha modificado la variedad de los ingredientes y la preparación de los alimentos, evolucionando hasta influir en cómo disfrutamos y nos comunicamos respecto a ellos. Del boca a boca se pasó a las publicaciones impresas, radio, televisión, internet y redes sociales. Hasta hace poco el protagonismo lo tenían los medios masivos tradicionales, especialmente la televisión y, en las zonas de mayor urbanización, gozaban de gran prestigio los medios impresos. En las grandes ciudades este apogeo lo han ido conquistando las plataformas digitales y redes sociales, pues cuanto más remotas las comunidades, menor es la penetración de los medios de comunicación. Las zonas rurales y aquellas con mayores niveles de pobreza son frecuentemente excluidas de esta evolución, existiendo aún comunidades que no disponen de cableado eléctrico o cobertura telefónica (Martínez Sosa, 2020).

La gastronomía se ha valido del medio audiovisual y ha facilitado el crecimiento de importantes figuras. En la televisión, se han destacado Eugenia Rojo, Deyanira Jackson, Jacqueline Henríquez, Wendy Gómez, Rosanna Ovalles, Wandy Robles y Leandro Díaz, entre otros. Han sido también producidos programas de temporada que resaltan ofertas gastronómicas particulares de una región o época del año, siendo ejemplo: "Chakall y Mangú", serie auspiciada por Nestlé que presentó aventuras del chef Chakall. A través de la plataforma Youtube, se encuentran: "Cocina Casera RD" con David Richardson Santana, "Cocinando con Ros Emely", "Simple by Clara", "Cocinando con Yolanda", "Chef Manu”, "Dominican Food TV", "El sazón de Mika".

Los periódicos (Diario Libre, Listín Diario, Hoy, El Caribe, El Día, etc.) con frecuencia incluyen artículos gastronómicos y, dentro de ellos, es común encontrar encartes en formato de revista o recetario coleccionable. Reconocidas marcas como Induveca, Rica, Maggi, Nestlé, Knorr, Supermercados Nacional y Supermercados La Cadena, los han utilizado para conectar con sus clientes y fomentar el consumo de determinados productos. Dentro de las revistas impresas, aún se edita Aldaba Gourmet, del Listín Diario y, ya fuera de circulación, fue destacable Gastroteca, editada por el chef Emil Vega y reconocida por su alta calidad. 


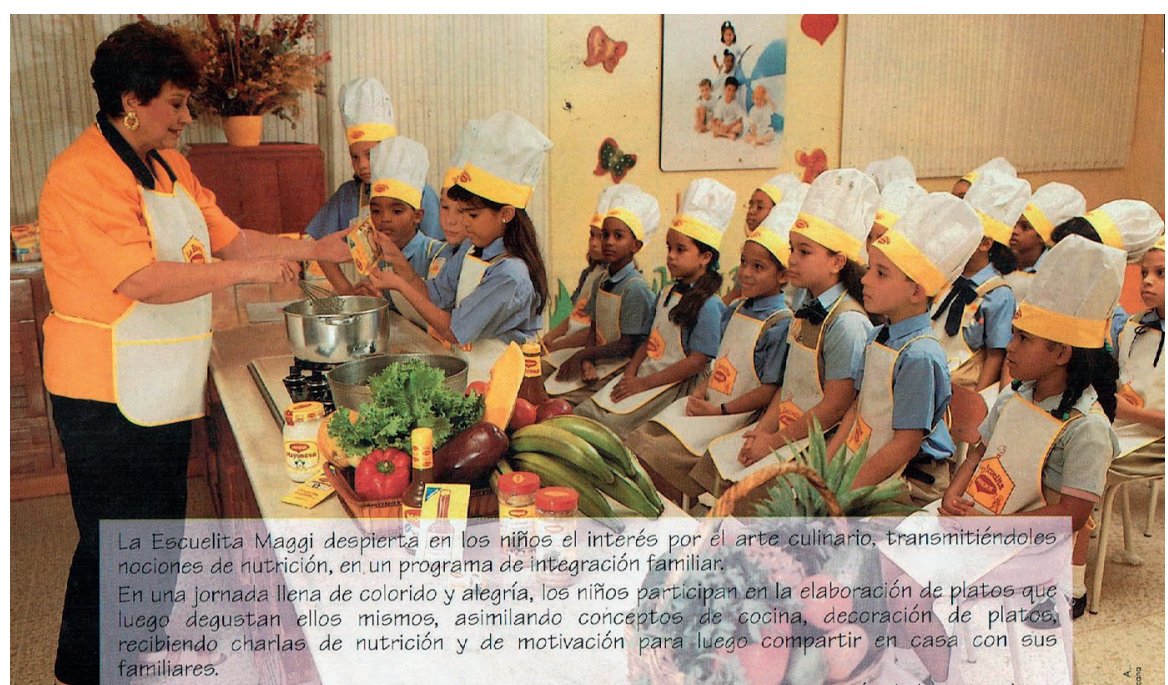

Figura 2. Imagen promocional de la Escuelita Maggi. Fuente: Calendario Maggi 1995.

Ciertos grupos empresariales, basándose en la promoción del patrimonio inmaterial dominicano, han apostado a la distribución y comercialización de productos de inspiración o elaboración local. Dentro de estas iniciativas de responsabilidad social corporativa, resulta admirable la labor realizada por el Grupo CCN a través de propuestas como: Orgullo de mi Tierra, Arte de Café y Corazón Dominicano (Martínez Sosa, 2020), y de Maggi, a través de sus recetarios y de la Escuelita Maggi, una iniciativa educativa que benefició varias generaciones.

\section{Resultados}

La creación de contenido gastronómico dominicano es cada vez mayor y se ha optado por abordarla a través de una investigación bibliográfica y una observación en medios sociales. El objetivo es identificar los perfiles de sujetos, marcas e iniciativas que fungen como embajadores digitales de dicha cultura culinaria, generando impacto en la sociedad.

\subsection{Producción bibliográfica}

Con frecuencia buscan investigar, informar, promover y actualizar el imaginario gastronómico dominicano. Cada autor lo hace desde su propia experiencia, mostrando influencias de su época, estética, escuela y técnica. En "Biblio-Hemerografía de la cultura tradicional y popular de la República Dominicana" (Pérez, 2009), se enlistan 89 piezas escritas sobre gastronomía criolla publicadas en libros, revistas y periódicos. Son el resultado de la producción intelectual de reconocidos nombres como Marcio Veloz Maggiolo, Dagoberto Tejeda Ortiz, Bernardo Vega, Fradique Lizardo, Carlos Esteban Deive, Manuel García Arévalo, Juan B. Nina, Carmenchu Brusíloff, Xiomarita Pérez y Nexcy D’ León, entre otros. 

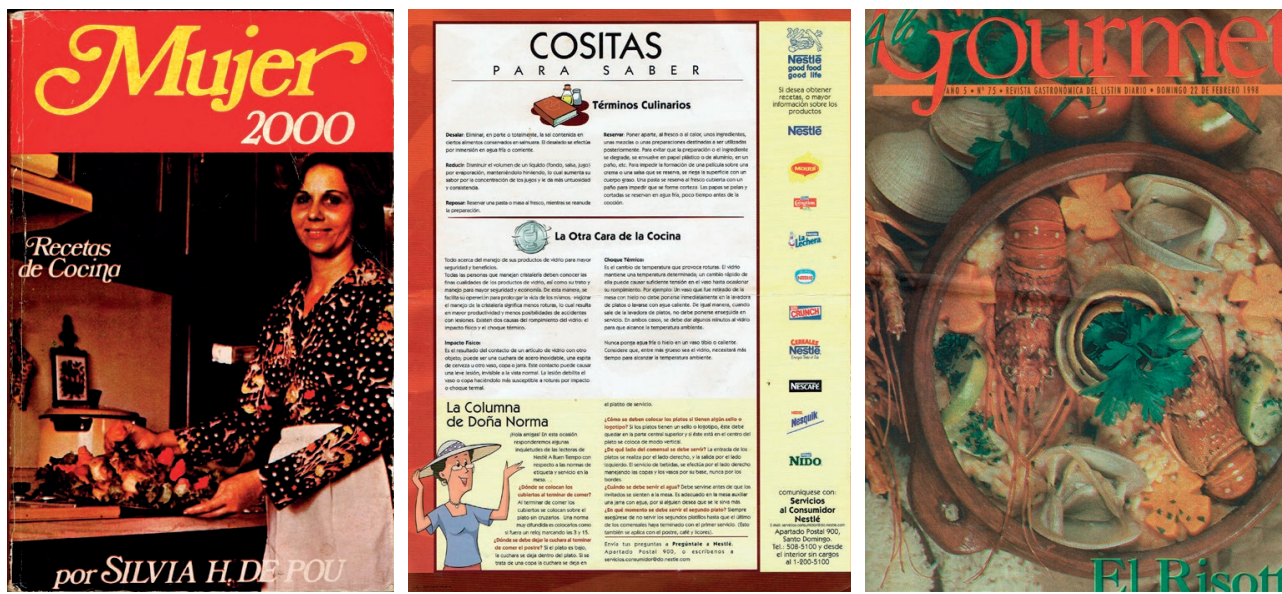

Figura 3. Publicaciones gastronómicas dominicanas, parte de una colección privada. Fuente: Biblioteca privada de Isis Mercedes Flaquer.

Para organizar estos hallazgos y presentar parte de la bibliografía gastronómica dominicana se creó una tabla. Más de 130 elementos fueron incluidos y organizados cronológicamente desde 1927 hasta este 2021, uniendo en una misma casilla los libros con varias ediciones o tomos, y los autores con varias publicaciones en un mismo año.

\section{Selección de la bibliografía gastronómica de la República Dominicana (1927-2021)}

Tabla 1. Bibliografía gastronómica de la República Dominicana(1927-2021), organizada por fecha, autory título.

\begin{tabular}{|c|c|c|}
\hline Fecha & Autor & Título \\
\hline 1927 & Ramón Emilio Jiménez & $\begin{array}{l}\text { Al amor del bohío. Tradiciones y costumbres } \\
\text { dominicanas }\end{array}$ \\
\hline 1938,1939 y 1940 & Amanda Ornes de Perelló & Manual de Economía Doméstica \\
\hline 1955 & Amanda Ornes de Perelló & Cocina Criolla \\
\hline 1959 & Ligia Vidal de Bornia & La Cocina Dominicana \\
\hline 1965 & Ligia Vidal de Bornia & Dominican Typical Meals / Comidas típicas dominicanas \\
\hline 1971 & Ligia Vidal de Bornia & La Cocina Ligia \\
\hline 1972 & Mercedes Amiama Tió & El arte de la cocina \\
\hline 1973 & Estrella Betances de Pujadas & Recetas Dominicanas \\
\hline 1976 & Guillermina González & Cocina dominicana y platos internacionales \\
\hline $1976,1980,2002$ y 2015 & Silvia Henríquez de Pou & Mujer 2000 \\
\hline 1976 & Gladys Chabebe de Cruzado & Primer libro de cocina dominicana publicado en EE.UU. \\
\hline 1977 & Ladies Guild of Santo Domingo & Recetas Dominicanas / Dominican Recipes \\
\hline 1979 & Adria Vidal de Mañón & Un tesoro de comidas completas \\
\hline 1981,1986 y 2007 & Miriam de Gautreaux & La magia del azúcar \\
\hline 1982 & Nilda Abreu de Suriel & Los menús de la Navidad \\
\hline
\end{tabular}




\begin{tabular}{|c|c|c|}
\hline Fecha & Autor & Título \\
\hline 1982 & Ladies Guild of Santo Domingo & Libro de Cocina / Cook Book \\
\hline 1983 & Esperanza de Lithgow & Cocina fácil \\
\hline 1984 & Estrella Betances de Pujada & Recetas dominicanas y del Caribe \\
\hline 1985 & Gaby Lehrer & Pollo Gourmet \\
\hline \multirow[t]{4}{*}{1988} & Licorera Brugal y Co. y José Chez & El ron en la historia dominicana \\
\hline & Checo & Vocabulario del ron \\
\hline & & 267 cócteles con Brugal \\
\hline & & Epigramas sobre el ron Brugal 1906-1911 \\
\hline 1988 & Valerie Grullón y Susan Pichardo & $\begin{array}{l}\text { A Taste of the Caribbean. Remembrances and Recipes } \\
\text { of the Dominican Republic }\end{array}$ \\
\hline 1991 & Mercedes Amiama Tió & 50 años de experiencia en el arte de la cocina \\
\hline 1992 & Ligia Vidal de Bornia & La Cocina Ligia en microondas \\
\hline 1993 & María Ramírez de Carías & La Cocina Dominicana \\
\hline 1996 & Yolanda Zouain de Castellanos & Mis 100 recetas con harina de maíz \\
\hline 1996 & $\begin{array}{l}\text { Marianela Castro, Luz María } \\
\text { Alcántara y Eunice Colón }\end{array}$ & Cocina dominicana \\
\hline 1996 & Bernardo Vega & Las frutas de los taínos \\
\hline 1997 & Yolanda Valdés de Del Monte & Hoy cocino yo \\
\hline 1998 & Kilma Canaán de Fernández & Cocinado en casa \\
\hline 1998 & Susana Byas & La cocina cocola de la tía Susana \\
\hline 1999 & José Ramón López & La alimentación y las razas \\
\hline 1999 & Elizabeth Sánchez & Consejos múltiples de cocina y algo más \\
\hline 1999 & Socorro Castellanos & Con los cinco sentidos. Las mejores recetas de Buenas \\
\hline & & Tardes a la Orden T.V. \\
\hline 1999 y 2006 & Juan B. Nina & El origen de la cocina dominicana \\
\hline 2000 & Elba Ysidora & Gourmet Fácil Festivo \\
\hline 2000 & Chea de Ortíz & Cocinando con Chea de Ortíz \\
\hline 2000 & Juan B. Nina & La cocina de las Américas \\
\hline 2001 y 2006 & Xilia Hernández & CocinArte \\
\hline 2002 & Mercasid & El arte de cocinar en casa \\
\hline 2003 & Roberto Cassá & $\begin{array}{l}\text { Raíces y desarrollo de un orgullo dominicano. Historia } \\
\text { de la cerveza en la República Dominicana }\end{array}$ \\
\hline 2003 & Claudia López & Cocinar es fácil \\
\hline 2004 & José Del Castillo & Agenda de fin de siglo \\
\hline 2004 & Eugenia Rojo & Recetas de la cocina de Eugenia Rojo \\
\hline 2004 & Chea de Ortiz (Mercedes Manzano) & Ensaladas, salsas y aderezos \\
\hline 2005 & Esperanza de Lithgow & Entremeses fáciles para ti \\
\hline 2005 & Chea de Ortiz (Mercedes Manzano) & Arroz para todos los gustos \\
\hline 2005 & Elba Ysidora & Gourmet Fácil. El pequeño gran libro de los sándwiches \\
\hline & & Gourmet Fácil. La Biblia de las salsas \\
\hline
\end{tabular}




\begin{tabular}{|c|c|c|}
\hline Fecha & Autor & Título \\
\hline 2005 & "Recetas de ayer y de hoy" & Recetas de ayer y de hoy \\
\hline 2005 & $\begin{array}{l}\text { Alejandro Paulino Ramos y Aquiles } \\
\text { Castro publican }\end{array}$ & Diccionario de cultura y folklore dominicano \\
\hline 2005 & Clara González e Ilana Benady & Aunt Clara's Dominican Cookbook \\
\hline 2006 & Eugenia Rojo & Cocina Cocina \\
\hline 2006 & Juan B. Nina & La cocina caribeña (historia y recetas) \\
\hline 2007 & Juan B. Nina & $\begin{array}{l}\text { Los dulces dominicanos (historia y recetas) } \\
\text { Folklore Gastronómico del Sur }\end{array}$ \\
\hline 2007 & Omar Paíno Perdomo & $\begin{array}{l}\text { Hongos comestibles de la República Dominicana. Guía } \\
\text { de campo }\end{array}$ \\
\hline 2007 & Brenda Gil & El arte del cocinao \\
\hline 2007 & Estela Aristy & Cocina criolla dominicana \\
\hline 2007 & Hortensia Sousa de Baquero & Recetas con sabor espiritual \\
\hline 2007 & Clara González e Ilana Benady & Traditional Dominican Cookery \\
\hline 2007 & $\begin{array}{l}\text { Marcio Veloz Maggiolo y Hugo } \\
\text { Tolentino Dipp }\end{array}$ & Gastronomía Dominicana. Historia del sabor criollo \\
\hline 2007 & Clarislandia Quiñones & Cocina Fácil Dominicana \\
\hline 2008 & $\begin{array}{l}\text { Clara Cabrera y Rosa Fiorinelli de } \\
\text { Albert }\end{array}$ & Cocina saludable \\
\hline 2008 & $\begin{array}{l}\text { Recopilación de } 31 \text { autores de los } \\
\text { que se encuentran Dolly Molinari } \\
\text { de Esteban, Margot Flaquer de } \\
\text { Sánchez y Sonia Saviñón de } \\
\text { Sánchez }\end{array}$ & Café de la leche. Nuestras recetas favoritas \\
\hline 2008 & Editora Corripio y Ana Alcántara & $\begin{array}{l}\text { My Mother's Kitchen. A Collection of Special Recipes by } \\
\text { Ana Alcántara \& Friends }\end{array}$ \\
\hline 2008 & Eugenia Rojo & El libro rojo de Eugenia \\
\hline 2008 & Juan B. Nina & $\begin{array}{l}\text { Bebidas Típicas Dominicanas } \\
\text { Diccionario Gastronómico Dominicano } \\
\text { Cócteles Dominicanos }\end{array}$ \\
\hline 2009 & Juan B. Nina & La cocina vegetariana dominicana \\
\hline 2009 & Mike Mercedes & Las recetas del maestro Mike Mercedes \\
\hline 2009 & Bismar Galán & $\begin{array}{l}\text { Sancocho dominicano. Persistencia y simbolismo de un } \\
\text { plato }\end{array}$ \\
\hline 2009 & Jacayaguila Carmona y Jorge Diep & 20 restaurantes gourmet y 20 diseñadores fashion \\
\hline 2009 & Jimmy Hungría & Gastronomía musical y bibliografías en construcción \\
\hline 2009 & Kin Sánchez & Nuestra verde navidad \\
\hline 2009 & Xilia Hernández & Mi amigo el arroz \\
\hline 2009 & Eugenia Rojo & Ligero y Gourmet \\
\hline 2010 & Delcy Mata & $\begin{array}{l}\text { Recetas dominicanas. El mágico secreto de la cocina } \\
\text { criolla }\end{array}$ \\
\hline
\end{tabular}




\begin{tabular}{|c|c|c|}
\hline Fecha & Autor & Título \\
\hline 2010 & José Rafael Sosa & Las Recetas del Doctor \\
\hline 2010 & Bismar Galán & $\begin{array}{l}\text { Sancocho dominicano: persistencia y simbolismo de un } \\
\text { plato }\end{array}$ \\
\hline 2011 & $\begin{array}{l}\text { Cruz Altagracia López Genao } \\
\text { "Yonki" }\end{array}$ & La magia de elaborar dulces \\
\hline 2011 & Zaida Lovatón de Sanz & 100 recetas y más de Doña Zaida \\
\hline 2011 & Adolfo Castañón & Conferencias Magistrales 2010 \\
\hline 2011,2013 y 2014 & Pasteurizadora Rica & Herencia Gastronómica Dominicana \\
\hline 2011 & Carmen Taveras & La cocina natural del nuevo siglo \\
\hline 2011 & Marisol Jorge & Cocina gourmet rápida \\
\hline 2011 & Sergio Ottato & El vino, Sócrates y yo \\
\hline 2011 & Xilia Hernández & $\begin{array}{l}\text { Postres y bebidas } \\
\text { Guarniciones y más } \\
\text { Carnes, aves, pescados, mariscos, panes y panecillos } \\
\text { Bocaditos, entradas, sopas, cremas y ensaladas }\end{array}$ \\
\hline 2012 & Benilda Llenas & Las bondades del plátano \\
\hline 2012 & $\begin{array}{l}\text { Vincenzina Egidi Balzarini (Doña } \\
\text { Enza) }\end{array}$ & Cocina Familiar de Doña Enza \\
\hline 2012 & Restaurante Porter House & Dominicana, la República de Sabores \\
\hline 2012 & Mari Núñez & Cómo Cocinar al Estilo Dominicano \\
\hline 2012 & Arturo Féliz-Camilo & Las recetas de Mamá Pura \\
\hline 2012 & Miriam de Gautreaux & Antología de la Repostería Dominicana. Siglos XX-XXI \\
\hline 2012 & Socorro Castellanos & El sabor de mi tierra \\
\hline 2013 & Juan B. Nina & $\begin{array}{l}\text { Los helados dominicanos } \\
\text { Folklore Gastronómico del Cibao }\end{array}$ \\
\hline 2013 & Arturo Féliz-Camilo & $\begin{array}{l}\text { Los mejores postres dominicanos } \\
\text { Diccionario Culinario Dominicano } \\
\text { El sazón de la cocina dominicana } \\
\text { Longaniza Dominicana, los secretos de la mejor lon- } \\
\text { ganiza del Caribe }\end{array}$ \\
\hline 2014 & Esperanza de Lithgow & La Olla Mágica de Esperanza Lithgow \\
\hline 2014 & Leandro Díaz & Cocinando sin ITBIS \\
\hline 2014 & Rosa María Gómez "La Chefa" & La Nueva Cocina Dominicana de La Chefa \\
\hline 2014 & Hugo Tolentino Dipp & Itinerario histórico de la gastronomía dominicana \\
\hline 2014,2016 y 2017 & Marivell Contreras & El sabor de las letras \\
\hline 2015 & Juan B. Nina & $\begin{array}{l}\text { Folklore Gastronómico del Este } \\
\text { El sabor del agua. Recetas de pescados y mariscos del } \\
\text { Caribe } \\
\text { Gastronomía de Cuaresma. Historia y recetas }\end{array}$ \\
\hline 2015 & Dominique Barkhausen & Eat, Love, Vita \\
\hline
\end{tabular}




\begin{tabular}{|c|c|c|}
\hline Fecha & Autor & Título \\
\hline 2016 & Juan B. Nina & Antropología de las frituras. Historia y recetas \\
\hline 2016 & Inés Páez (Chef Tita) & La Nueva Cocina Dominicana \\
\hline 2016 & Annie Camacho & Mis dulces tentaciones \\
\hline 2016 & Fundación Sabores Dominicanos & $\begin{array}{l}\text { Identificando la esencia y los matices de nuestros } \\
\text { sabores }\end{array}$ \\
\hline 2017 & Juan B. Nina & $\begin{array}{l}\text { Historia de la cocina taína } \\
\text { Herencia africana en la gastronomía dominicana. } \\
\text { Historia y recetas }\end{array}$ \\
\hline 2017 & María Marte & Soñar, luchar, cocinar \\
\hline 2017 & Francisco de Padua Morales & El mangú \\
\hline 2017 & Doña Rosa Fiorinelli de Albert & La Cocina de Rossette \\
\hline 2017 & Arturo Feliz Camilo & $\begin{array}{l}\text { Longaniza Dominicana: Los secretos de la mejor } \\
\text { Longaniza del Caribe }\end{array}$ \\
\hline 2018 & Inicia (recopilación de autores) & Sabores Ancestrales \\
\hline 2019 & Arturo Feliz Camilo & $\begin{array}{l}\text { La Magia de Unas Habichuelas Sabrosas: Habichuelas } \\
\text { Dominicanas }\end{array}$ \\
\hline 2020 & Sagrario Hernández & $\begin{array}{l}\text { Recetas Dominicanas: } 10 \text { recetas que debes probar en } \\
\text { esta cuarentena }\end{array}$ \\
\hline 2021 & $\begin{array}{l}\text { Clara González, Verónica Cervera, } \\
\text { Erica Dinho, Alejandra Graf y Layla } \\
\text { Pujol }\end{array}$ & Yo cocino latino \\
\hline 2021 & Esperanza Lithgow & Entremeses Fáciles Para Ti \\
\hline
\end{tabular}

Fuente: Elaboración propia. Basado en Hungría (2018) y Pérez (2009).

Interesantes libros extranjeros resaltan recetas preferidas de figuras dominicanas con proyección internacional: "The Metropolian Opera Cookbook" (Bondagree, 1988) muestra platos del laureado diseñador Oscar de la Renta; el libro "Jazz Cooks" (Young y Stankus, 1992) incluye la versión del cerdo asado dominicano con arroz del reconocido músico Michel Camilo. Para los deportistas "Diamond Dishes: From the Kitchens of Baseball's Biggest Stars" (Loria, 2011) muestra los gustos de dominicanos en las Grandes Ligas del Béisbol.

\subsection{Identificación de perfiles}

La evolución en materia de comunicación y estilos periodísticos ha sido influida por el surgimiento de nuevos medios digitales como: blogs, Facebook, Instagram, Twitter, Google Maps, Tik Tok y otros, en los cuales se promueve y aprovecha el contenido generado por los usuarios. Se aprecia un cada vez más minucioso cuidado por los detalles y, en algunos casos, altísimas frecuencias de publicación. Se apuesta a cautivar de manera visual y escrita con estímulos que despiertan el interés del receptor, atrapando su imaginación y deseo. Se observa también una reducción de costos en herramientas especializadas como hielo falso y stands, circunstancias que han permitido que hoy la comunicación culinaria tenga un ecosistema más avanzado y complejo. A raíz del escenario descrito han surgido nuevos perfiles como: 
Foodie: persona que muestra un gran interés en el disfrute de la comida. El término fue acuñado luego de que en 1984 el New York Times publicara el artículo "British Find Food Need Not Be Stodgy", luego de un banquete celebrado en Londres por el lanzamiento del libro "The Official Foodie Handbook" de Ann Barr y Paul Levy, en que se describe el concepto foodie. Hoy vemos en redes sociales a foodies que salen en búsqueda de las nuevas ofertas gastronómicas y luchan por ser los primeros en mostrar sus hallazgos.

Food Stylist (Styling): se dedican al arte del "estilismo de alimentos" y, junto al chef, captan la esencia de los platos y representan visualmente sus sabores. En una producción audiovisual hacen que el producto se vea lo más estilizado y apetecible posible. Buscan estimular la compra y captar la atención de la audiencia con emplatados creativos, ingeniosas herramientas y trucos. Dentro de sus retos está la popular publicidad engañosa (Molins, 2015), pues al incentivar el consumo de alimentos cuidando los detalles de manera extrema, a veces la apariencia del resultado dista de la preparación habitual. Hay restaurantes que contratan food stylists para que velen porque los platos transmitan las mismas emociones que atrajeron al comensal.

Chefs: el Diccionario de la Real Academia de la Lengua Española lo define como una voz tomada del francés chef ('jefe'), que se emplea en español con los sentidos de "jefe de cocina de un restaurante" y, especialmente, cocinero profesional de grandes dotes y reconocido prestigio. Actualmente, academias especializadas ofrecen carreras en el área gastronómica y títulos de cocineros con distintos niveles en la jerarquía de la cocina, como los chefs y su asistente, el "sous chef" o "segundo jefe".

Periodistas culinarios (gastronómicos): se dedican al estudio, recopilación e investigación del sector gastronómico, velando por su difusión y promoción a través de los medios. Requiere estar al día con las tendencias e innovaciones de recetas, técnicas, restaurantes, chefs, etc. Puede recaer en diferentes géneros del periodismo, teniendo gran fuerza los críticos que se enfocan en la interpretación de la propuesta gastronómica y proceden a su calificación (Universidad San Francisco de Quito, 2021). Inicia su auge en República Dominicana hacia 2016.

La popularización de algunos de estos términos es reciente y el tamaño de las producciones en las cuales suelen verse involucrados puede variar desde pequeños proyectos hasta enormes campañas de marketing. Para hacer una producción comunicacional culinaria transmedia, por ejemplo, pueden intervenir directamente 5 roles: el chef que prepara el plato, el food stylist que lo estiliza, el fotógrafo experto en iluminación y en captar la esencia de cada propuesta, el foodie que comparte información, y el periodista culinario que difunde esta información en medios de comunicación tradicionales y digitales.

Con el crecimiento de la industria alimentaria, la comunicación gastronómica se ha convertido en un pilar financiero que da a conocer y promocionar este popular elemento de una cultura, mientras genera retribución económica. Por ello, la gastronomía es una de las principales áreas de la Economía Naranja, que abarca actividades de carácter cultural que producen bienes y servicios. Para contribuir con el desarrollo de la misma, se han habilitado múltiples capacitaciones y proyectos. Sirven de ejemplo las inversiones realizadas a la Industria de La Piña, el Proyecto de Mapeo de la Gastronomía, la Escuela Gastronómica Típica en Samaná y la Fábrica Artesanal del Conconete Dominicano (Sabores Dominicanos, 2016). Las capacitaciones, por su parte, han abarcado las áreas de crítica gastronómica 


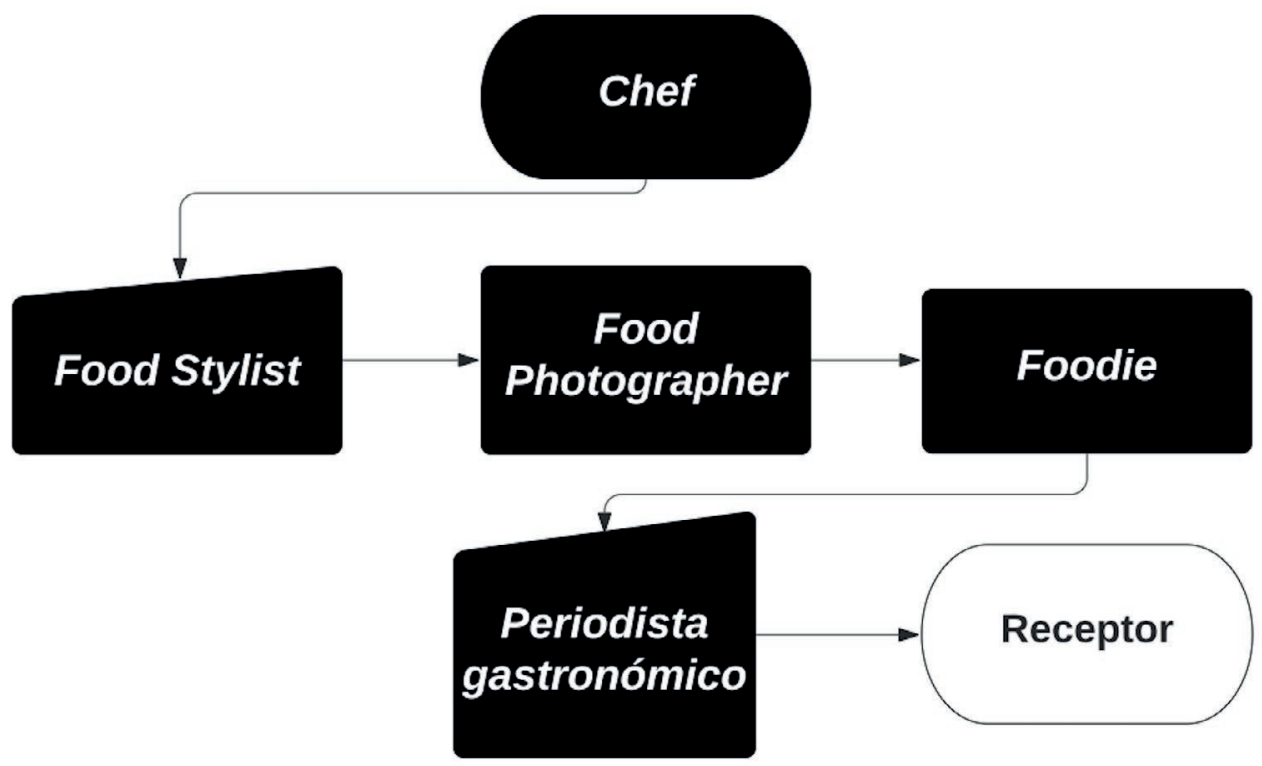

Figura 3. Flujograma de roles que pueden intervenir en una producción comunicacional gastronómica. Fuente: Elaboración propia.

y Gastro Ficción, además del diseño de un Bachillerato Técnico en modalidad Servicios Gastronómicos. Estas iniciativas son comunicadas a través de eventos como "La gastronomía y el periodismo para hacer marca del país".

\subsection{Embajadores Gastronómicos Digitales}

Para la selección de los integrantes del siguiente listado de foodies, chefs y periodistas gastronómicos, se consideró lo siguiente:

- Actividad semanal, tomando como medio Instagram: frecuencia en la cual se mantiene activa la cuenta.

- Cantidad de seguidores: número de seguidores que les gusta y apoyan el contenido.

- Calidad de las publicaciones: manera en la cual las publicaciones realizadas enganchan al receptor.

- Engagement de seguidores: interacciones de los seguidores con la cuenta.

- Premios y reconocimientos: galardones que aumentan la confianza e interés del cliente.

- Publicaciones Impresas: ayuda visual para apreciar de otra manera las imágenes e información. 
Chefs, foodies y periodistas gastronómicos relevantes en República Dominicana, 2021.

Tabla 2. Chefs, foodies y periodistas gastronómicos relevantes en República Dominicana, 2021.

\begin{tabular}{|c|c|c|}
\hline Categoría & Nombre & Link de Instagram \\
\hline \multirow[t]{10}{*}{ Foodies } & Bocao & https://www.instagram.com/bocao/ \\
\hline & Rincones & https://www.instagram.com/rincones_rd/ \\
\hline & Burger Bastards & https://www.instagram.com/burgerbastards/ \\
\hline & TheFoodLifeDR & https://www.instagram.com/thefoodlifeofdr/ \\
\hline & FitfoodiesRD & https://www.instagram.com/fitfoodiesrd/ \\
\hline & Vaina de Flaca & https://www.instagram.com/vainadeflaca/ \\
\hline & AlacartadorRD & https://www.instagram.com/alacartadord/ \\
\hline & Vaina de Gordo & https://www.instagram.com/vainadegordo/ \\
\hline & TheBurgerCatador & https://www.instagram.com/theburgercatador/ \\
\hline & TasteltRD & https://www.instagram.com/tasteitrd/ \\
\hline \multirow[t]{19}{*}{ Chef } & Jacqueline Henríquez & https://www.instagram.com/jacquelinchefrd/ \\
\hline & Nikol Morillo & https://www.instagram.com/nikolmorillo/ \\
\hline & Leandro Díaz & https://www.instagram.com/chefleandrodiaz/ \\
\hline & Carolina Arias & https://www.instagram.com/chefcarolinaarias \\
\hline & María Marte & https://www.instagram.com/chefmariamarte/ \\
\hline & Keily Manuel Busby E. & https://www.instagram.com/kmbusbye/ \\
\hline & Eliana Mateo & https://www.instagram.com/elianamateo/ \\
\hline & Gina Vicini & https://www.instagram.com/ginavicini/ \\
\hline & Manuel Méndez & https://www.instagram.com/chefmanurd/ \\
\hline & Cristina M. Báez & https://www.instagram.com/cris_baez/ \\
\hline & José Manuel Lombardero & https://www.instagram.com/josemlombardero/ \\
\hline & Julissa Pieter & https://www.instagram.com/chefjulird/ \\
\hline & Chef Tita & https://www.instagram.com/lacheftita/ \\
\hline & Paulette Tejada & https://www.instagram.com/paulettetejada/ \\
\hline & Haydée Salcedo Objío "Chef Dede" & https://www.instagram.com/chefdederd/ \\
\hline & Francis Pena & https://www.instagram.com/francispenachef/ \\
\hline & Erik Malmsten & https://www.instagram.com/cheferikmalmsten/ \\
\hline & Yuri (Chef Pandita) & https://www.instagram.com/chefpandita/ \\
\hline & Laura Amelia & https://www.instagram.com/lauraamalialabaker/ \\
\hline \multirow{4}{*}{$\begin{array}{l}\text { Periodismo } \\
\text { Gastronómico y } \\
\text { Foodie }\end{array}$} & RutaGourmetDO & https://www.instagram.com/rutagourmetdo/ \\
\hline & Ivonne Soriano (Paladar de paseo) & https://www.instagram.com/paladardepaseo/ \\
\hline & Juan de Dios Valentín (Foodie and Traveler DR) & https://www.instagram.com/foodieandtravelerdr/ \\
\hline & Elaine Hernández (Bocatips) & https://www.instagram.com/bocatips/ \\
\hline
\end{tabular}

Fuente: Elaboración propia. 


\subsection{Otras iniciativas}

En el transcurso de esta investigación fue evidente que la bibliografía y los perfiles presentados son solo una fracción del amplio y dinámico espectro gastronómico dominicano, y se entiende relevante mencionar las siguientes iniciativas:

\section{- Día Nacional de la Gastronomía Dominicana}

El 2018 fue especial para la cocina y gastronomía dominicana pues fue reconocida con el nombramiento de un día dedicado para su celebración. A partir de ese año, el segundo domingo de diciembre es el "Día Nacional de la Cocina y Gastronomía Dominicana". Este decreto, aprobado por la cámara de Diputados, es la Ley 20-18 de la Constitución. La iniciativa para la creación de este día parte de rescatar los platos de nuestro país que puede que hayan quedado en el olvido, al igual que hacer sobresalir aquellos que nos caracterizan de manera internacional.

\section{- Premio Nacional a la Gastronomía Dominicana}

La Asociación Nacional de Gastronomía y Hostelería (AGH) es la encargada anualmente de organizar la gala de Premios Nacionales a la Gastronomía Dominicana, donde se hace reconocimientos a los restaurantes, chefs e iniciativas más importantes del año. Sus categorías premiadas son "Categoría Premio del Público", "Categoría Premio a la Iniciativa en la Gastronomía”, "Categoría Premio a la Excelencia Gastronómica”, "Categoría Premio Marca País”.

- Televisión: Mazola Academy y Master Chef

Las competencias de reality TV tienen producciones de alto nivel en escenografía y diseño, sin embargo, se les da más énfasis a los concursantes que a la comida. La pionera nacional en
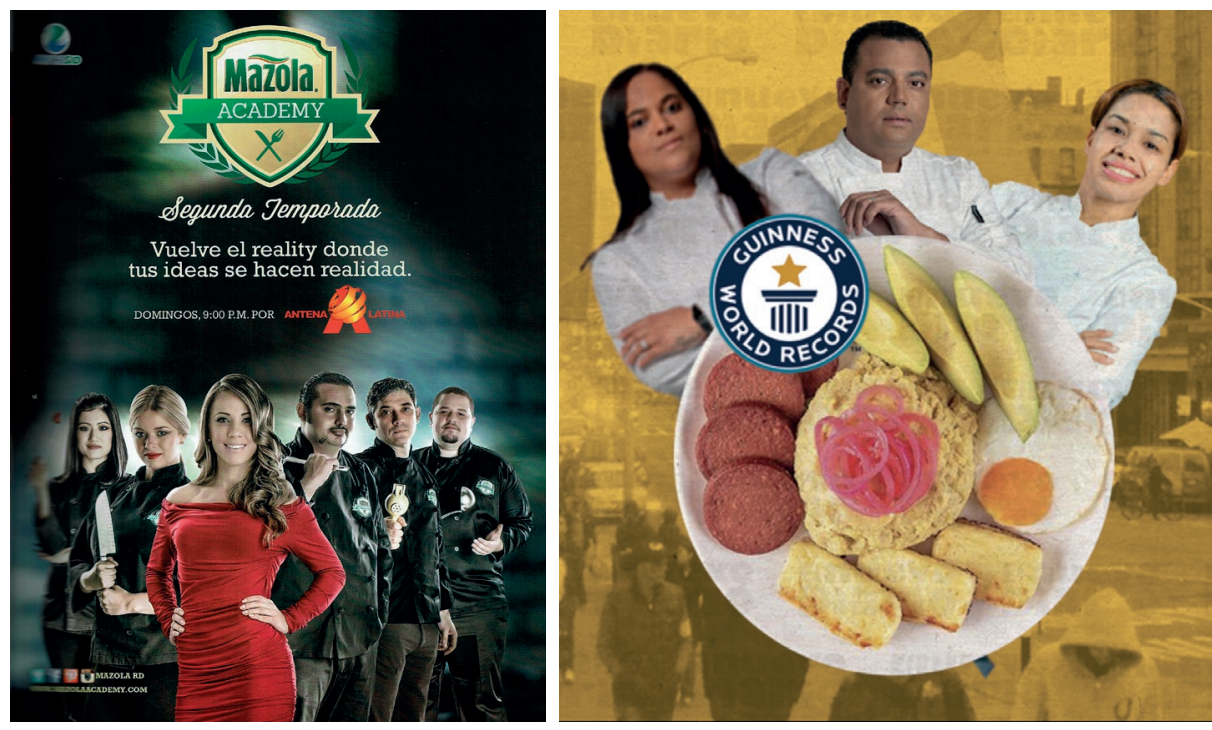

Figura 5. Imágenes promocionales de Mazola Academy y del Premio Guinness al Mangú más grande del mundo.

Fuente: el Súper y El Nuevo Diario. 
este formato fue Mazola Academy, que data de 2012. Allí, 18 integrantes elegidos por jueces competían en cada episodio formando equipos en los que se buscaba impulsar el talento y la creatividad gastronómica dominicana, y que eran evaluados tanto en sabor como en estilismo. Cuando llegó por primera Masterchef Dominicana, 3 mil personas asistieron a postularse y 3 millones de dólares se invirtieron en el estudio de 17,700 pies cuadrados que posicionó a la República Dominicana a la vanguardia de la televisión regional. En 2021 este programa ya tiene tres modalidades diferentes: "Masterchef", "Masterchef Junior", "Masterchef Celebrity".

\section{- Museografía y organización de información: Mapeo gastronómico}

Uno de los beneficiados de la segunda Convocatoria Nacional de Proyectos Culturales fue la iniciativa del mapeo gastronómico liderada por la gestora cultural Olga Valdez y la chef Dévaki Pratt. El portal RutaGourmet.do afirmó que este "hizo un levantamiento de informaciones en las 10 macro regiones de nuestra geografía nacional, estableciendo un marco teórico específico para cada una, con el objetivo de rescatar recetas, técnicas, instrumentos y/o costumbres existentes en cada provincia del país". Esta iniciativa presentó también una exposición multisensorial en Ágora Mall en agosto de 2016, incluyó presencia en redes sociales y una página web que, a la fecha, está inactiva.

\section{- Fundación Sabores Dominicanos}

Organización sin ánimo de lucro cuyo objetivo es desarrollar, impulsar e implementar proyectos para la valorización y desarrollo del sistema gastronómico dominicano, con el fin de posicionar al país a nivel internacional. Crearon en el 2015 la Propuesta Gastronómica RD 20-20, iniciativa que buscaba la evolución de la cultura culinaria y que fue implementada por el impulso que genera la gastronomía al turismo y a las fuentes de trabajo. A través de este proyecto nació el Portal Gastronómico Dominicano, espacio multifuncional para la difusión y promoción de la cocina tradicional criolla. Esta Fundación es también responsable de la iniciativa Premios Nacionales de Periodismo y Literatura Gastronómica que impulsa investigación, valoración y difusión de la literatura gastronómica dominicana. Por su parte el proyecto de Salvaguarda Acervo Culinario Dominicano une a un equipo de investigadores y cocineros que realiza inventarios de recetas de platos y bebidas dominicanos con el fin de preservar esta rama del patrimonio dominicano.

\section{- Record Guiness: Mangú}

La República Dominicana en distintas ocasiones se ha propuesto romper un récord mundial para entrar en el aclamado libro de los Récords Guinness. En el 2017 el programa Despierta América y la Comunidad Dominicana en Nueva York crearon la versión más grande registrada de uno de los platos criollos más icónicos: el mangú. En esa época se alcanzó un peso promedio de 645 libras de plátano verde, cebolla encurtida, salami, queso y huevos fritos. La dedicación de seguir expandiendo los límites de nuestra gastronomía hizo que, el 1 ro de octubre de 2021 y con un total de 1,000 libras, la diáspora dominicana volviera a romper el récord del mangú más grande del mundo esta vez moldeado con la forma del país y con plátanos obtenidos en Tamayo, provincia Bahoruco. Los encargados de coordinar la hazaña fueron los chefs María Marte, Amilkar y Pamela Gonell en el marco del evento Flavor Fusion Fest.

\section{- Semana Gastronómica Digital}

Los periodistas y foodies Paladar de Paseo y Foodie and Traveler DR, crearon el concepto de la Semana de la Gastronomía Dominicana Digital donde llevaron a las redes sociales un 
homenaje a la historia y al desarrollo de la cocina dominicana, presentando aportes de grandes chefs como Leandro Díaz, Manuel Méndez (Chef Manu), Martín Omar, Chef July, Noemí Díaz, Jacqueline Henríquez, Rodolfo Mesa (Chef Ramch), La Chefa Gómez, Chef Tita, Deyanira Jackson, Le Chef Francisco, Carolina Arias, Odalís Rodríguez, Arturo Feliz y Clara González.

\section{- Creación y promoción de Rutas Gastronómicas}

Apreciando el valor que puede llevar a las comunidades, han surgido diversas iniciativas para consolidar rutas e itinerarios de turismo gastronómico. Estos invitan a probar platos y sabores icónicos, mientras se conocen las tradiciones, empresas e instituciones detrás de los mismos. Entre estas se destacan las Rutas: de la Miel, del Casabe, del Banano y del Chivo, que forman parte del "Proyecto de Fortalecimiento del Mecanismo de Desarrollo del Turismo Sostenible Basado en la Comunidad en la Región Norte, República Dominicana". Este proyecto ha sido implementado por el Ministerio de Turismo (MITUR) y el Instituto Nacional de Formación Técnico Profesional (INFOTEP) en coordinación con el Ministerio de Economía, Planificación y Desarrollo (MEPyD), y bajo la asistencia técnica y financiera de la Agencia de Cooperación Internacional del Japón (JICA).

\section{Conclusiones}

La gastronomía es una extensión de la cultura que lleva tantos años de desarrollo como el ser humano mismo. Esta ha sufrido incontables cambios tanto en su registro de sabores como en las formas en que estos se obtienen, experimentan y comunican. Mientras el tratamiento de la imagen y la narrativa utilizadas evidencian los avances, tendencias y cánones de cada época, el aumento y democratización de las tecnologías ha favorecido el surgimiento de un ecosistema mucho más avanzado y complejo.

Tras haber iniciado la identificación de perfiles de abanderados y embajadores digitales e ilustrado la evolución de la comunicación de la gastronomía en la República Dominicana. Se deja abierta la invitación a que se continúe esta línea de investigación, a que se mapee el ecosistema comunicacional dominicano en materia culinaria, evaluando a profundidad las herramientas, plataformas y sujetos que se van abriendo camino en esta área en base a experimentación, innovación y creatividad.

Los nuevos roles y formas de interactuar, difundir y promocionar la gastronomía criolla tienen a su alcance estrategias multiplataforma y la posibilidad aprovechar al máximo la comunicación transmedia. Esto, sumado a la creciente popularidad de la Economía Naranja, presenta un panorama esperanzador en el que sea posible aprovechar la gastronomía para alimentar el cuerpo, conectar comunidades, potenciar sus capacidades de desarrollo y engrandecer la calidad culinaria de las naciones.

\section{Referencias}

BENCOS, A. (2020). Fundación Sabores Dom: Importancia de su labor. <https://silohubierasabido. wordpress.com/2020/01/13/fundación-sabores-dom-importancia-de-su-labor/> [Consulta: 9 de octubre de 2021]. 
DELGADO MERADON, P. (2019). Gastronomía dominicana. Historia del sabor criollo (2 de 2) <https:// www.elcaribe.com.do/gente/cultura/apuntes-de-infraestructura/gastronomia-dominicanahistoria-del-sabor-criollo-2-de-2/> [Consulta: 9 de octubre de 2021].

DELGADO MERADON, P. (2019). Gastronomía dominicana. Historia del sabor criollo (1 de 2). <https:// www.elcaribe.com.do/gente/cultura/apuntes-de-infraestructura/gastronomia-dominicanahistoria-del-sabor-criollo-1-de-2/> [Consulta: 9 de octubre de 2021].

HUNGRÍA, J. (2018). "Apuntes para una bibliografía gastronómica dominicana”. En: Acento. <https:// acento.com.do/opinion/apuntes-una-bibliografia-gastronomica-dominicana-1-2-8600794.html> [Consulta: 15 de octubre de 2021].

TOLENTINO DIPP, H. (2018). Apuntes en torno al itinerario histórico de la gastronomía dominicana <http://saboresdominicanos.org/incia-saludable/apuntes-en-torno-al-itinerario-historico-de-lagastronomia-dominicana> [Consulta: 8 de octubre de 2021].

SANCHEZ DESCHAMPS, B. SUAZO REYES, L y ARAUJO BRITO, E. (2016). Impacto de la Oferta Folklórica y Gastronómica del Restaurante JALAO como Atracción Turística Complementaria de la Ciudad Colonial de Santo Domingo. <https://bibliotecaunapec.blob.core.windows.net/tesis/TESIS_ Cl_ATH_04_2016_ET170190.pdf> [Consulta: 8 de octubre de 2021].

CAMPOS, J. (2016). "Artesanos de la gastronomía dominicana". <https://listindiario.com/ la-vida/2021/03/21/662101/artesanos-de-la-gastronomia-dominicanas.

RUTA GOURMET DO (2016). "Mapeo de la Gastronomía Dominicana" cierra con exposición. <https:// rutagourmet.do/mapeo-de-la-gastronomia-dominicana/>.

FLORENTINO, V. (2016). "De la cocina a la TV”. El Caribe.

PÉREZ, X. (2009). Biblio-Hemerografía de la cultura tradicional y popular de la República Dominicana. República Dominicana: Editora Amigo del Hogar.

PORTILLO, M. (2014). "Listado de libros de cocina dominicanos" cierra con exposición. < [http://recetadeliciosa.blogspot.com/2014/10/listado-de-libros-de-cocina-dominicanos.html/].

EL CARIBE. (2021). Mangú dominicano establece Record Guinness en New York. <[https://www. elcaribe.com.do/gente/estilo/dominicanos-romperan-record-guinness-con-el-mangu/].

ZABALA, J. (2017). Dominicanos romperán Record Guinness con el mangú. <[https://do.municipiosaldia. com/ultramar/item/27451-mangú-dominicano-establece-record-guinness-en-new-york/].

ASONAHONORES (2018). Diputados convierten en Ley "Día Nacional de la Cocina y Gastronomía Dominicana". < <https://www.asonahores.com/diputados-convierten-en-ley-dia-nacional-de-lacocina-y-gastronomia-dominicana/]>.

MINISTERIO DE EDUCACIÓN DE LA REPÚBLICA DOMINICANA (2018). Bachiller Técnico en servicios gastronómicos. < <https://www.ministeriodeeducacion.gob.do/docs/direccion-de-educaciontecnico-profesional/BFcT-tuh001-3-bt-servicios-gastronomicospdf.pdf]>.

INSTITUCIONAL DOMINICANA (2018).Periodismo gastronómico en The Foodie Studies. < [http://www. institucionaldominicana.com/periodismo-gastronomico-the-foodie-studies/]>. 
Maricha Martínez Sosa, Lucero Liriano, Georgina Batista Schrils

NODAL CULTURAL (2015). Periodismo gastronómico en The Foodie Studies. < [https://www. nodalcultura.am/2016/02/cultura-y-gastronomia-dominicanas/>.

VELASCO, M. USFQ (2021).Periodismo Gastronómico: ¿Qué Es Y Para Qué Sirve? < [https://noticias. usfq.edu.ec/2021/10/periodismo-gastronomico-que-es-y-para.html>.

LARROUSECOCINA (2020). El Chef de cocina: su origen y su historia. < [https://laroussecocina.mx/ nota/el-chef-de-cocina-su-origen-y-su-historia/]>

REAL ACADEMIA ESPAÑOLA. Definición de chef. <[https://dle.rae.es/chef/]> 